TRANSACTIONS OF THE

AMERICAN MATHEMATICAL SOCIETY

Volume 363, Number 4, April 2011, Pages 2191-2209

S 0002-9947(2010)05214-6

Article electronically published on October 28, 2010

\title{
MULTIPLICATIVE $q$-HYPERGEOMETRIC SERIES ARISING FROM REAL QUADRATIC FIELDS
}

\author{
KATHRIN BRINGMANN AND BEN KANE
}

\begin{abstract}
Andrews, Dyson, and Hickerson showed that $2 q$-hypergeometric series, going back to Ramanujan, are related to real quadratic fields, which explains interesting properties of their Fourier coefficients. There is also an interesting relation of such series to automorphic forms. Here we construct more such examples arising from interesting combinatorial statistics.
\end{abstract}

\section{INTRODUCTION AND STATEMENT OF RESULTS}

Andrews, Dyson and Hickerson [3] intensively studied the function

$$
\sigma(q):=1+\sum_{n=1}^{\infty} \frac{q^{\frac{n(n+1)}{2}}}{(-q)_{n}}=1+q-q^{2}+2 q^{3}+\cdots+4 q^{45}+\ldots
$$

which first appeared in Ramanujan's lost notebook 18. Here we define as usual $(a)_{n}=(a ; q)_{n}:=\prod_{j=0}^{n-1}\left(1-a q^{j}\right)$. In [3], the authors obtain an exact formula for the coefficients of (1.1) by relating this function to the arithmetic of $\mathbb{Q}(\sqrt{6})$. This implies that the coefficients have multiplicative properties, $\sigma$ is lacunary, i.e. its coefficients are almost always zero, and yet attains every integer infinitely many times. In subsequent work a few more such examples were found by Lovejoy, Corson et al. and others, building on known Bailey pairs, which led to interesting applications (see for example [5, 9, 12, 13]). Additional to these properties the function $\sigma$ has a natural combinatorial interpretation as the number of partitions into distinct parts with even rank minus those with odd rank. Recall that Dyson's rank of a partition [11] is defined as its largest part minus its number of parts.

The function $\sigma$ is in several ways related to automorphic forms. First, using Hecke $L$-series, Cohen [8] related this function to classical Maass forms. Second, if instead of partitions into distinct parts one considers unrestricted partitions, one obtains Ramanujan's mock theta function

$$
f(q):=1+\sum_{n=1}^{\infty} \frac{q^{n^{2}}}{(-q)_{n}^{2}},
$$

which is, due to recent work of Zwegers [19] and Bringmann-Ono [6, 7], known to be the holomorphic part of a harmonic Maass form, as are all the rank generating functions. Harmonic Maass forms are generalizations of modular forms, in that

Received by the editors December 23, 2008 and, in revised form, September 27, 2009.

2000 Mathematics Subject Classification. Primary 11P81, 11E16, 05A17.

The first author was partially supported by NSF grant DMS-0757907 and the Alfried-Krupp prize.

(C)2010 American Mathematical Society Reverts to public domain 28 years from publication 
they satisfy the same transformation law, and (weak) growth conditions at cusps, but instead of being holomorphic, they are annihilated by the weight $k$ hyperbolic Laplacian. To describe a third way in which $\sigma$ is related to automorphic forms, we recall from [3] that the main step in relating $\sigma$ to $\mathbb{Q}(\sqrt{6})$ is to prove the following representation of $\sigma$ as Hecke-type sums:

$$
\sigma(q)=\sum_{\substack{n \geq 0 \\|j| \leq n}}(-1)^{n+j} q^{\frac{n(3 n+1)}{2}-j^{2}}\left(1-q^{2 n+1}\right) .
$$

We note that this representation can also be viewed as a "false mock theta function". To be more precise, using work of Zwegers [19, the function

$$
\sum_{\substack{n \geq 0 \\|j| \leq n}}(-1)^{n+j} q^{\frac{n(3 n+1)}{2}-j^{2}}\left(1+q^{2 n+1}\right)
$$

can be viewed as the "holomorphic part" of an indefinite theta series and is related to one of Ramanujan's sixth order mock theta functions.

In this paper we find more examples resembling (1.1), which have interesting combinatorial interpretations and which we relate to the real quadratic fields $\mathbb{Q}(\sqrt{2})$ and $\mathbb{Q}(\sqrt{3})$. Of particular interest are Theorems 1.1 and 1.2. since for its proof we find two new Bailey pairs (see Theorem 2.3) which are of independent interest.

We first consider $q$-hypergeometric series related to $K=\mathbb{Q}(\sqrt{2})$ and denote as usual with $\mathcal{N}(\mathbf{a})$ the norm of an ideal a in $O_{K}$, the ring of integers of $K$.

Theorem 1.1. The function

$f_{1}(q):=\sum_{n=0}^{\infty} \frac{q^{\frac{n^{2}+n}{2}}}{(-q)_{n}\left(1-q^{2 n+1}\right)}=1+2 q+3 q^{3}+q^{5}+2 q^{6}+2 q^{7}+4 q^{10}+\cdots+6 q^{52}+\ldots$ satisfies

$$
q f_{1}\left(q^{16}\right)=\sum_{\mathcal{N}(a) \equiv 1} q^{\mathcal{N}(a)}
$$

Let us next describe how the function $f_{1}(q)$ naturally arises in the theory of partitions. To do so, consider

$$
f_{1}(x ; q):=\frac{1}{1-q}+\sum_{n=1}^{\infty} \frac{q^{\frac{n^{2}+n}{2}}}{(x q)_{n}\left(1-x^{2} q^{2 n+1}\right)},
$$

for which we have $f_{1}(q)=f_{1}(-1 ; q)$. We will now construct a set of partitions $P_{1}$ and a statistic $r_{1}(\lambda)$ for which, as we will show in Subsection 4.1, the coefficient of $x^{r} q^{m}$ in $f_{1}(x ; q)-q$ will be precisely the number of partitions $\lambda \in P_{1}$ of $|\lambda|=m$ with $r_{1}(\lambda)=r$. Then the $m$-th coefficient of $f_{1}(q)-q$ will clearly give the number of partitions $\lambda \in P_{1}$ of $|\lambda|=m$ with $r_{1}(\lambda)$ even minus the number of those with $r_{1}(\lambda)$ odd.

Let $P_{1}$ be the set of partitions with the following properties: One is the only part which may be repeated, and if it is repeated, say occurring $r+1$ times, then the second smallest part (if it exists) is at least $2 r$ and the third smallest part (if it exists) is at least two more than the second smallest part. For $\lambda \in P_{1}$, let $r_{1}(\lambda)$ denote the rank of $\lambda$ if $\lambda$ has no repeated parts of size 1 and otherwise $r_{1}(\lambda)$ is the rank of $\lambda$ without its parts of size 1 . 
Theorem 1.2. The function

$f_{2}(q):=\sum_{n=1}^{\infty} \frac{q^{\frac{n^{2}+n}{2}}}{(-q)_{n-1}\left(1-q^{2 n-1}\right)}=q+q^{2}+2 q^{3}+2 q^{5}+2 q^{6}+\cdots+4 q^{14}+\cdots+3 q^{77}+\ldots$

satisfies

$$
q^{-7} f_{2}\left(q^{16}\right)=\sum_{\mathcal{N}(a) \equiv 9} q^{\mathcal{N}(a)}
$$

The partition theoretic interpretation of $f_{2}(q)$ has striking similarities to that of $\sigma(q)$. Define $P_{2}$ to be the set of partitions into distinct parts restricted by the following conditions: The rank of $\lambda \in P_{2}$ is at least $2(s-1)$, where $s$ is the smallest part, and the second smallest part is at least $2 s$. In Subsection 4.2, we show that $f_{2}(q)$ is the generating function for $\lambda \in P_{2}$ with $r_{2}(\lambda)$ even minus those with $r_{2}(\lambda)$ odd, where $r_{2}(\lambda)$ is the rank if there is more than one part and $r_{2}(\lambda)=0$ otherwise. We develop this similarly to the case of $f_{1}$ by defining $f_{2}(x ; q)$ in Subsection 4.2 and taking $x=-1$, where $x$ will be used to count the statistic $r_{2}(\lambda)$. We will suppress such definitions for the remainder of the introduction.

Theorem 1.3. The function

$$
f_{3}(q):=\sum_{n=0}^{\infty} \frac{(q)_{2 n}}{(-q)_{2 n+1}} q^{n}=1-2 q^{3}+q^{4}+2 q^{8}-2 q^{11}+\cdots+3 q^{24}+\ldots
$$

satisfies

$$
q f_{3}\left(q^{2}\right)=\sum_{\boldsymbol{a} \subset O_{K}}\left(\frac{-4}{\mathcal{N}(\boldsymbol{a})}\right) q^{\mathcal{N}(a)}
$$

where $\left(\frac{c}{d}\right)$ denotes the Kronecker symbol.

To relate $f_{3}$ to partitions, we recall the notion of overpartitions and overpartition pairs. An overpartition [10] of $\mathrm{n}$ is a partition of $\mathrm{n}$ in which the first occurrence of a number can be overlined. An overpartition pair [14] of $\mathrm{n}$ is a pair of overpartitions $(\mu, \lambda)$, where the sum of all the parts is n. Here we consider $P_{3}$ as the set of overpartitions pairs $\Lambda=(\mu, \lambda)$ with the following restrictions on $\mu$ and $\lambda$ : If the largest part of $\mu$ is overlined, then it must also occur non-overlined and if $\mu$ is the empty partition, then no parts of $\lambda$ may be overlined. Moreover, if $\lambda$ has any parts, then its largest part is exactly one greater than the largest part of $\mu$, parts of $\lambda$ of size one less than its largest part cannot be overlined, and the number of occurrences of the largest part in $\lambda$ is at least half of all parts in $\lambda$ (with strict inequality if the largest part of $\lambda$ is overlined). In Subsection 4.3 we show that $f_{3}(q)$ is the generating function for $\Lambda \in P_{3}$ with $r_{3}(\Lambda)$ odd minus those with $r_{3}(\Lambda)$ even, where $r_{3}(\Lambda)$ counts the number of occurrences of the largest part in $\lambda$ minus the number of parts in $\mu$.

Theorem 1.4. The function

$$
f_{4}(q):=\sum_{n=0}^{\infty} \frac{(q)_{2 n+1}}{(-q)_{2 n+2}} q^{n+1}=q-q^{2}-q^{4}+2 q^{7}-q^{8}+q^{9}-2 q^{14}+\cdots-3 q^{98}+\ldots
$$


satisfies

$$
f_{4}(q)=-\sum_{a \subset O_{K}}(-1)^{\mathcal{N}(a)} q^{\mathcal{N}(a)} .
$$

The combinatorial interpretation of $f_{4}$ is similar to that of $f_{3}$. We let $P_{4}$ be the set of overpartition pairs $\Lambda=(\mu, \lambda)$ with the following restrictions on $\mu$ and $\lambda$ : If the largest part of $\mu$ is overlined, then it must also occur non-overlined. Moreover, if $\lambda$ has any parts, then its largest part equals the largest part of $\mu$, the largest part of $\lambda$ cannot be overlined, $\lambda$ has an even number of parts, and the number of occurrences of the largest part in $\lambda$ is at least half of the total number of its parts. In Subsection 4.4 we show that $f_{4}(q)$ is the generating function for $\Lambda \in P_{4}$ with $r_{4}(\lambda)$ odd minus those with $r_{4}(\lambda)$ even, where $r_{4}(\lambda)$ is half the number of parts of $\lambda$ minus the number of parts of $\mu$.

We next turn to the real quadratic field $L:=\mathbb{Q}(\sqrt{3})$.

Theorem 1.5. The function

$$
f_{5}(q):=\sum_{n=0}^{\infty}(-1)^{n} \frac{(q)_{n}}{\left(q ; q^{2}\right)_{n+1}} q^{\frac{n^{2}+n}{2}}=1+q^{2}+2 q^{3}+q^{6}+2 q^{8}+\cdots+3 q^{42}+\ldots
$$

satisfies

$$
q f_{5}\left(q^{4}\right)=\sum_{\substack{\boldsymbol{a} \subset O_{L} \\ \mathcal{N}(\boldsymbol{a}) \equiv 1}} q^{\mathcal{N}(\boldsymbol{a})} .
$$

To see how $f_{5}$ can be viewed in the framework of partitions, we require some notation. For a partition $\lambda=\left(\lambda_{1}, \ldots, \lambda_{n}\right)$ into $n$ distinct parts (in decreasing order), define the sequence $\ell_{i}:=\lambda_{i}-\lambda_{i+1}(1 \leq i \leq n-1)$ and $\ell_{n}:=\lambda_{n}$. Also, define $E:=E_{\lambda}:=\left\{2 \leq r \leq n: \ell_{r}\right.$ is even $\}$ and $e:=\# E$. Let $P_{5}$ be the set of partitions $\lambda$ into distinct parts with the following properties: If $\lambda$ has only a single part, then this part is not congruent to 1 modulo 3 , and otherwise $\ell_{1} \geq d_{\lambda}+1$ and $\ell_{1} \equiv d_{\lambda}+1(\bmod 3)$, where $d_{\lambda}$ is defined in Subsection 4.5. In Subsection 4.5, we show that $f_{5}(q)$ is the generating function for partitions $\lambda \in P_{5}$ with $r_{5}(\lambda)$ odd minus those with $r_{5}(\lambda)$ even, where $r_{5}(\lambda):=\lambda_{2}$, if the partitions contains at least two parts and $r_{5}(\lambda):=1$ otherwise.

Remark. We note that the $q$-hypergeometric series

$$
\sum_{n=0}^{\infty} \frac{(q)_{n}}{\left(q ; q^{2}\right)_{n+1}} q^{\frac{n^{2}+n}{2}}=1+2 q+q^{2}+2 q^{3}+2 q^{4}+3 q^{6}+\cdots+6 q^{81}+\ldots,
$$

which is obtained from $f_{5}(q)$ by deleting the $(-1)^{n}$, is the modular form $\frac{\eta^{4}(2 \tau)}{\eta^{2}(\tau)}$ which is related to $\mathbb{Q}(i)$.

Theorem 1.6. The function

$$
f_{6}(q):=\sum_{n=1}^{\infty}(-1)^{n} \frac{\left(q^{2} ; q^{2}\right)_{n-1}}{\left(q^{n}\right)_{n}} q^{n}=-q-2 q^{3}-2 q^{6}-q^{7}-2 q^{10}-\cdots-4 q^{36}-\ldots
$$

satisfies

$$
q^{-1} f_{6}\left(q^{4}\right)=-\sum_{\substack{\boldsymbol{\alpha}(\boldsymbol{a}) \equiv 3 \\(\bmod 4)}} q^{\mathcal{N}(a)}
$$


Let $P_{6}$ be the set of overpartitions $\lambda$ with the following properties: The largest part cannot be overlined, every overlined part must also occur non-overlined, and the number of repetitions of the largest part plus the number of overlined parts is greater than half of all of the parts. In Subsection 4.6, we show that $f_{6}(q)$ is the generating function for $\lambda \in P_{6}$ with $r_{6}(\lambda)$ even minus those with $r_{6}(\lambda)$ odd, where $r_{6}(\lambda)$ counts the largest part minus the number of overlined parts.

Theorem 1.7. The function

$f_{7}(q):=\sum_{n=0}^{\infty}(-1)^{n} \frac{q^{n^{2}+n}\left(q^{2} ; q^{2}\right)_{n}}{(-q)_{2 n+1}}=1-q+2 q^{4}-q^{5}-2 q^{7}+q^{8}+\cdots+3 q^{40}+\ldots$ satisfies

$$
q f_{7}\left(q^{3}\right)=-\sum_{\mathcal{N}(a) \equiv 1 O_{L}(\bmod 3)}(-1)^{\mathcal{N}(a)} q^{\mathcal{N}(\mathbf{a})} .
$$

To interpret $f_{7}$, we denote for an overpartition $\lambda$ by $M(\lambda)$ the number of times that the largest part occurs if the largest part is greater than one, and set $M(\lambda)=0$ otherwise. Let $P_{7}$ be the set of overpartitions with the following properties: If the largest part is greater than one, then the largest part equals the number of nonoverlined parts plus one, the second largest part size is at most $M(\lambda)+1$, and only parts of size less than or equal to $M(\lambda)$ may be overlined. In Subsection 4.7, we show that $f_{7}(q)$ is the generating function for $\lambda \in P_{7}$ with an even number of parts minus those with an odd number of parts.

Theorem 1.8. The function

$$
f_{8}(q):=\sum_{n=1}^{\infty} \frac{(q)_{n-1}}{\left(-q^{n}\right)_{n}} q^{n}=q+q^{3}-2 q^{4}-2 q^{8}+2 q^{9}+\cdots-4 q^{48}+\cdots+3 q^{81}+\ldots
$$

satisfies

$$
q^{-1} f_{8}\left(q^{3}\right)=\sum_{\mathcal{N}(a) \equiv 2}(-1)^{\mathcal{N}(a)} q^{\mathcal{N}(a)} .
$$

Let $P_{8}$ be the set of overpartitions with the following properties: The largest part cannot be overlined and the number of repetitions of the largest part size is greater than half of all non-overlined parts. We set $r_{8}(\lambda)$ to be the number of repetitions of the largest part minus the number of overlined parts. In Subsection 4.8, we show that $f_{8}$ is the generating function for overpartitions $\lambda \in P_{8}$ with an $r_{8}(\lambda)$ minus those with $r_{8}(\lambda)$ even.

We next use the arithmetic of $K$ and $L$ to determine properties for the functions $f_{i}$ which resemble those of $\sigma$.

Corollary 1.9. The functions $f_{i}$ are lacunary.

We let

$S_{i}:=\left\{m \in \mathbb{Z}\right.$, there are infinitely many $n$ such that $a_{f_{i}}(n)=m$, where $a_{f_{i}}(n)$ denotes the $n$-th coefficient of $f_{i}$. 
Corollary 1.10. We have

$$
S_{i}= \begin{cases}\mathbb{N}_{0} & \text { if } i \in\{1,2,5\} \\ -\mathbb{N}_{0} & \text { if } i=6, \\ \mathbb{Z} & \text { if } i \in\{4,7\} \\ \mathbb{N}_{0} \cup-2 \mathbb{N} & \text { if } i \in\{3,8\}\end{cases}
$$

It would be interesting to further investigate the functions $f_{i}$ recovered here, for example relating them to harmonic Maass forms as described above for $\sigma$. In particular the combinatorics of $f_{2}$ resemble those of $\sigma$. So the question arises, whether one can construct new mock theta functions by considering unrestricted partitions instead of partitions into distinct parts. Furthermore $\sigma$ also occurred in interesting number theoretical identities involving sums of tails of $\eta$-quotients (see for example [5, 16]). It would be interesting to investigate whether our functions $f_{i}$ play related roles. We plan to address these questions in future research.

The paper is organized as follows. Section 2 is devoted to establishing two new Bailey pairs required for the proofs of Theorems 1.1 and 1.2 which are of independent interest. In Section 3 we write the functions $f_{i}$ as Hecke-type sums and establish our main theorems. In Section 4 we prove the natural connection of the functions $f_{i}$ to the above described partition statistics.

\section{TWO NEW BAILEY PAIRS}

Here we establish two new Bailey pairs required for the proofs of Theorems 1.1 and 1.2. Let us first recall the definition of a Bailey pair and Bailey's Lemma. For details and background on Bailey pairs, we refer the reader to Chapter 3 of [2].

Definition 2.1. Two sequences $\left\{\alpha_{n}\right\}$ and $\left\{\beta_{n}\right\}$ form a Bailey pair relative to $a$ if for all $n \geq 0$, we have

$$
\beta_{n}=\sum_{r=0}^{n} \frac{\alpha_{r}}{(q)_{n-r}(a q)_{n+r}} .
$$

Moreover, if only the $\beta_{n}$ are given, then $\alpha_{n}$ can be determined using Bailey inversion:

$$
\alpha_{n}=\left(1-a q^{2 n}\right) \sum_{j=0}^{n} \frac{(a q)_{n+j-1}(-1)^{n-j} q^{\left(\frac{n-j}{2}\right)}}{(q)_{n-j}} \beta_{j} .
$$

To establish our main theorems we will use a limiting case of Bailey's Lemma.

Lemma 2.2. If $\alpha_{n}$ and $\beta_{n}$ form a Bailey pair relative to $a$, then we have, providing both sides converge absolutely,

$$
\sum_{n=0}^{\infty} \frac{\left(\rho_{1}, \rho_{2}\right)_{n}}{\left(\frac{a q}{\rho_{1}}, \frac{a q}{\rho_{2}}\right)_{n}}\left(\frac{a q}{\rho_{1} \rho_{2}}\right)^{n} \alpha_{n}=\frac{\left(a q, \frac{a q}{\rho_{1} \rho_{2}}\right)_{\infty}}{\left(\frac{a q}{\rho_{1}}, \frac{a q}{\rho_{2}}\right)_{\infty}} \sum_{n=0}^{\infty}\left(\rho_{1}, \rho_{2}\right)_{n}\left(\frac{a q}{\rho_{1} \rho_{2}}\right)^{n} \beta_{n}
$$

where $\left(a_{1}, \ldots, a_{r} ; q\right)_{n}=\left(a_{1}, \ldots, a_{r}\right)_{n}:=\prod_{j=1}^{r}\left(a_{j}\right)_{n}$.

We show the following. 
Theorem 2.3. (1) The sequences $a_{n}, b_{n}$ form a Bailey pair relative to $a=1$ :

$$
\begin{aligned}
b_{0}=b_{0}(q) & :=0, \\
b_{n}=b_{n}(q) & :=\frac{(-1)^{n}\left(q ; q^{2}\right)_{n-1}}{(q)_{2 n-1}}, \\
a_{2 n}=a_{2 n}(q) & :=\left(1-q^{4 n}\right) q^{2 n^{2}-2 n} \sum_{j=-n}^{n-1} q^{-2 j^{2}-2 j}, \\
a_{2 n+1}=a_{2 n+1}(q) & :=-\left(1-q^{4 n+2}\right) q^{2 n^{2}} \sum_{|j| \leq n} q^{-2 j^{2}} .
\end{aligned}
$$

(2) The sequences $\alpha_{n}, \beta_{n}$ form a Bailey pair relative to $a=q$ :

$$
\begin{aligned}
& \beta_{n}=\beta_{n}(q):=\frac{(-1)^{n}\left(q ; q^{2}\right)_{n}}{(q)_{2 n+1}} \\
& \alpha_{2 n}=\alpha_{2 n}(q):=\frac{1}{1-q}\left(q^{2 n^{2}+2 n} \sum_{-n \leq j \leq n-1} q^{-2 j^{2}-2 j}+q^{2 n^{2}} \sum_{|j| \leq n} q^{-2 j^{2}}\right) \\
& \alpha_{2 n+1}=\alpha_{2 n+1}(q):=-\frac{1}{1-q}\left(q^{2 n^{2}+4 n+2} \sum_{|j| \leq n} q^{-2 j^{2}}\right. \\
&\left.+q^{2 n^{2}+2 n} \sum_{-n-1 \leq j \leq n} q^{-2 j^{2}-2 j}\right)
\end{aligned}
$$

To facilitate the proof of the theorem, we first define and then evaluate the sum

$$
U_{n}:=(-1)^{n} \sum_{j=1}^{n}\left[\begin{array}{c}
n+j-1 \\
2 j-1
\end{array}\right] q^{\left(\begin{array}{c}
n-j \\
2
\end{array}\right)}\left(q ; q^{2}\right)_{j-1}
$$

where $\left[\begin{array}{l}n \\ j\end{array}\right]:=\frac{(q)_{n}}{(q)_{j}(q)_{n-j}}$.

Proposition 2.4. We have for $n \geq 0$ :

$$
\begin{aligned}
U_{2 n} & =q^{2 n^{2}-2 n} \sum_{j=-n}^{n-1} q^{-2 j^{2}-2 j}, \\
U_{2 n+1} & =-q^{2 n^{2}} \sum_{|j| \leq n} q^{-2 j^{2}} .
\end{aligned}
$$

Proof. It is easy to see that both sides of Proposition 2.4 satisfy $U_{0}=0$ and $U_{1}=-1$. To finish the proof it is enough to show that (2.2) satisfies the recurrence

$$
U_{n+2}=q^{2 n} U_{n}+2(-1)^{n} .
$$

To prove (2.3), we let

$$
V_{n}:=(-1)^{n}\left(U_{n+2}-q^{2 n} U_{n}\right)
$$


Inserting (2.2) yields

$$
\begin{aligned}
V_{n}= & \sum_{j=1}^{n+2} \frac{(q)_{n+1+j} q^{\frac{1}{2}(n+2-j)(n+1-j)}}{(q)_{2 j-1}(q)_{n-j+2}}\left(q ; q^{2}\right)_{j-1} \\
& -\sum_{j=1}^{n} \frac{(q)_{n-1+j} q^{\frac{1}{2}(n-j)(n-1-j)+2 n}}{(q)_{2 j-1}(q)_{n-j}}\left(q ; q^{2}\right)_{j-1} \\
= & q \sum_{j=1}^{n+2} \frac{(q)_{n+j-1}\left(q ; q^{2}\right)_{j}}{(q)_{2 j-1}(q)_{n-j+2}}\left(1-q^{2 n+2}\right) q^{\frac{1}{2}(n-j)^{2}+\frac{3 n}{2}-\frac{3 j}{2}}
\end{aligned}
$$

Using

$$
\begin{aligned}
(q)_{n+j-1} & =(q)_{n-1}\left(q^{n}\right)_{j} \\
(q)_{n+2-j} & =\frac{(q)_{n+2}}{q^{(n+2) j} q^{-\frac{j(j-1)}{2}}(-1)^{j}\left(q^{-n-2}\right)_{j}}
\end{aligned}
$$

we obtain

$$
V_{n}=\frac{q^{1+\frac{n^{2}}{2}+\frac{3 n}{2}}\left(1+q^{n+1}\right)}{\left(1-q^{n}\right)\left(1-q^{n+2}\right)} \sum_{j=1}^{\infty} \frac{(-1)^{j}\left(q^{n}, q^{-n-2}\right)_{j} q^{j}}{(-q, q)_{j-1}} .
$$

We next recall the following transformation which is due to Heine (see (2.6) of [2]):

$$
\sum_{n=0}^{\infty} \frac{(a, b)_{n}}{(q, c)_{n}}\left(\frac{c}{a b}\right)^{n}=\frac{\left(\frac{c}{a}, \frac{c}{b}\right)_{\infty}}{\left(c, \frac{c}{a b}\right)_{\infty}} .
$$

We use (2.4) with $a=q^{n+1}, b=q^{-n-1}$, and $c=-q$, yielding

$$
V_{n}=\frac{q^{\frac{n^{2}}{2}+\frac{n}{2}}\left(-q^{-n},-q^{n+2}\right)_{\infty}\left(1+q^{n+1}\right)}{(-q,-q)_{\infty}} .
$$

Then $V_{n}=2$ follows from the identities

$$
\begin{aligned}
\left(-q^{-n}\right)_{\infty} & =2 q^{-\frac{n(n+1)}{2}}(-q)_{n}(-q)_{\infty} \\
\left(-q^{n+2}\right)_{\infty} & =\frac{(-q)_{\infty}}{(-q)_{n+1}} .
\end{aligned}
$$

This proves (2.3) and thus Proposition 2.4.

Proof of Theorem 2.3. We now show Theorem 2.3 (1). Using the Bailey inversion (2.1) with $a=1$, we have

$$
\begin{aligned}
a_{n} & =\left(1-q^{2 n}\right) \sum_{j=0}^{n} \frac{(q)_{n+j-1}}{(q)_{n-j}}(-1)^{n-j} q^{\left(\begin{array}{c}
n-j \\
2
\end{array}\right)} b_{j} \\
& =\left(1-q^{2 n}\right) \sum_{j=1}^{n} \frac{(q)_{n+j-1}}{(q)_{n-j}(q)_{2 j-1}}\left(q ; q^{2}\right)_{j-1}(-1)^{n} q^{\left(\begin{array}{c}
n-j \\
2
\end{array}\right)} \\
& =\left(1-q^{2 n}\right)(-1)^{n} \sum_{j=1}^{n}\left[\begin{array}{c}
n+j-1 \\
2 j-1
\end{array}\right]\left(q ; q^{2}\right)_{j-1} q^{\left(\begin{array}{c}
n-j \\
2
\end{array}\right)} \\
& =\left(1-q^{2 n}\right) U_{n} .
\end{aligned}
$$

This directly gives (1) using Proposition 2.4. 
We next turn to the proof of Theorem 2.3 (2) and use (2.1) with $a=q$ to obtain

$$
\begin{aligned}
\alpha_{n} & =\frac{1-q^{2 n+1}}{1-q} \sum_{j=0}^{n} \frac{(q)_{n+j}}{(q)_{n-j}}(-1)^{n+j} q^{\left(\begin{array}{c}
n-j \\
2
\end{array}\right)} \beta_{j} \\
& =\frac{1-q^{2 n+1}}{1-q}(-1)^{n} \sum_{j=0}^{n} \frac{(q)_{n+j}\left(q ; q^{2}\right)_{j}}{(q)_{n-j}(q)_{2 j+1}} q^{\left(\begin{array}{c}
n-j \\
2
\end{array}\right)} \\
& =\frac{1}{1-q}(-1)^{n} \sum_{j=0}^{n}\left[\begin{array}{c}
n+j+1 \\
n-j
\end{array}\right] \frac{1-q^{2 n+1}}{1-q^{n+j+1}} q^{\left(\begin{array}{c}
n-j \\
2
\end{array}\right)}\left(q ; q^{2}\right)_{j} .
\end{aligned}
$$

Writing

$$
1-q^{2 n+1}=1-q^{n+j+1}+q^{n+j+1}\left(1-q^{n-j}\right)
$$

yields

$$
\begin{aligned}
\alpha_{n}= & \frac{1}{1-q}(-1)^{n}\left(\sum_{j=0}^{n}\left[\begin{array}{c}
n+j+1 \\
n-j
\end{array}\right] q^{\left(\frac{n-j}{2}\right)}\left(q ; q^{2}\right)_{j}\right. \\
& \quad+\sum_{j=0}^{n-1}\left[\begin{array}{c}
n+j \\
n-j-1
\end{array}\right] q^{\left.\left(\frac{n-j}{2}\right) q^{n+j+1}\left(q ; q^{2}\right)_{j}\right)} \\
= & \frac{1}{1-q}\left(-U_{n+1}+q^{2 n} U_{n}\right) .
\end{aligned}
$$

Substituting $U_{n}$ from Proposition 2.4 gives the desired equality for $\alpha_{n}$.

\section{Proofs of the main theorems}

The main step in the proof of Theorem 1.1 is to rewrite $f_{1}$ as a Hecke-type sum, which relies on the Bailey pair obtained in Theorem 2.3 (2).

Proposition 3.1. We have

$$
f_{1}(q)=\sum_{\substack{n \geq 0 \\-n-1 \leq j \leq n}} q^{4 n^{2}+5 n+1-2 j^{2}-2 j}\left(1+q^{6 n+6}\right)+\sum_{\substack{n \geq 0 \\-n \leq j \leq n}} q^{4 n^{2}+n-2 j^{2}}\left(1+q^{6 n+3}\right) .
$$

Proof. We use the Bailey pair from Theorem 2.3 (2) in Bailey's Lemma with $\rho_{1} \rightarrow$ $\infty$ and $\rho_{2}=q$. Using the fact that

$$
\lim _{\rho \rightarrow \infty} \frac{(\rho)_{n}}{\rho^{n}}=(-1)^{n} q^{\frac{n(n-1)}{2}},
$$

the " $\beta$-side" of Bailey's Lemma equals

$$
\frac{1}{1-q} \sum_{n=0}^{\infty} \frac{\left(q ; q^{2}\right)_{n}(q)_{n} q^{\frac{n(n+1)}{2}}}{(q)_{2 n+1}}=\frac{1}{1-q} \sum_{n=0}^{\infty} \frac{q^{\frac{n(n+1)}{2}}}{(-q)_{n}\left(1-q^{2 n+1}\right)}=\frac{1}{1-q} f_{1}(q) .
$$

The " $\alpha$-side" equals

$$
\sum_{n=0}^{\infty} q^{n(2 n+1)} \alpha_{2 n}-\sum_{n=0}^{\infty} q^{(2 n+1)(n+1)} \alpha_{2 n+1} .
$$

Plugging in $\alpha_{n}$ from Theorem 2.3 (2) and multiplying by $1-q$ yields Proposition 3.1 .

We have now developed the necessary pieces to show Theorem 1.1 
Proof of Theorem 1.1. To finish the proof of Theorem 1.1 we first observe that Propositon 3.1 yields

$$
\begin{aligned}
q f_{1}\left(q^{16}\right)= & \sum_{\substack{n \geq 0 \\
-n-1 \leq j \leq n}}\left(q^{(8 n+5)^{2}-2(4 j+2)^{2}}+q^{(8 n+11)^{2}-2(4 j+2)^{2}}\right) \\
& +\sum_{\substack{n \geq 0 \\
|j| \leq n}}\left(q^{(8 n+1)^{2}-2(4 j)^{2}}+q^{(8 n+7)^{2}-2(4 j)^{2}}\right) .
\end{aligned}
$$

We next use Lemma 3 of [3] and unique factorization in $O_{K}$ to rewrite each ideal a occurring in equation (1.2) uniquely as $\mathbf{a}=(u+v \sqrt{2})$ with $u>0$ and $-\frac{1}{2} u<v \leq$ $\frac{1}{2} u$. The congruence condition $\mathcal{N}(\mathbf{a})=u^{2}-2 v^{2} \equiv 1(\bmod 16)$ corresponds to the four summands occurring in equation (3.1). This completes the proof of Theorem 1.1 .

As in the case of $f_{1}$, the main step in the proof of each of our main theorems will be to use Bailey's Lemma to first rewrite the function as a Hecke-type sum. The proposition below gives the desired Hecke-type sum for $f_{2}$.

Proposition 3.2. We have

$$
f_{2}(q)=\sum_{\substack{n \geq 1 \\-n \leq j \leq n-1}}\left(1+q^{2 n}\right) q^{4 n^{2}-n-2 j^{2}-2 j}+\sum_{\substack{n \geq 0 \\|j| \leq n}} q^{4 n^{2}+3 n+1-2 j^{2}}\left(1+q^{2 n+1}\right) .
$$

Proof. We use the Bailey pair from Theorem 2.3 (1) in Bailey's Lemma with $\rho_{1} \rightarrow$ $\infty$. We divide both sides by $1-\rho_{2}$, and then let $\rho_{2} \rightarrow 1$. After this the " $\beta$-side" equals

$$
\sum_{n \geq 1} \frac{(q)_{n-1}\left(q ; q^{2}\right)_{n-1}}{(q)_{2 n-1}} q^{\frac{n^{2}+n}{2}}=\sum_{n \geq 1} \frac{(q)_{2 n-2}}{(-q)_{n-1}(q)_{2 n-1}} q^{\frac{n^{2}+n}{2}}=f_{2}(q) .
$$

On the " $\alpha$-side", we use equation (2.5) to obtain

$$
\sum_{n=1}^{\infty} \frac{(-1)^{n} q^{\frac{n^{2}+n}{2}}}{1-q^{n}} a_{n}=\sum_{n=1}^{\infty} q^{2 n^{2}+n}\left(1+q^{2 n}\right) U_{2 n}-\sum_{n=0}^{\infty} q^{2 n^{2}+3 n+1}\left(1+q^{2 n+1}\right) U_{2 n+1} .
$$

Inserting Proposition 2.4 proves Proposition 3.2

Proof of Theorem 1.2. Proposition 3.2 gives

$$
\begin{aligned}
q^{-7} f_{2}\left(q^{16}\right)= & \sum_{\substack{n \geq 1 \\
-n \leq j \leq n-1}}\left(q^{(8 n-1)^{2}-2(4 j+2)^{2}}+q^{(8 n+1)^{2}-2(4 j+2)^{2}}\right) \\
& +\sum_{\substack{n \geq 0 \\
|j| \leq n}}\left(q^{(8 n+3)^{2}-2(4 j)^{2}}+q^{(8 n+5)^{2}-2(4 j)^{2}}\right) .
\end{aligned}
$$

We write $\mathbf{a}$ in (1.3) as in the proof of Theorem 1.1. The condition $\mathcal{N}(\mathbf{a}) \equiv 9$ $(\bmod 16)$ translates into the four summands of (3.2), proving Theorem 1.2 .

We recall the following Bailey pair from Theorem 2.3 of [4, which will play a pivotal role in the proof of Theorem 1.3 . 
Theorem 3.3. The following $A_{n}^{\prime}$ and $B_{n}^{\prime}$ form a Bailey pair relative to a:

$$
A_{n}^{\prime}=A_{n}^{\prime}(q):=\frac{q^{n^{2}}(b c)^{n}\left(1-a q^{2 n}\right)(a / b, a / c)_{n}}{(1-a)(b q, c q)_{n}} \sum_{j=0}^{n} \frac{(-1)^{j}\left(1-a q^{2 j-1}\right)(a)_{j-1}(b, c)_{j}}{q^{j(j-1) / 2}(b c)^{j}(q, a / b, a / c)_{j}}
$$

and

$$
B_{n}^{\prime}=B_{n}^{\prime}(q):=\frac{1}{(b q, c q)_{n}} .
$$

The main step of the proof of Theorem 1.3 is given in the following proposition.

Proposition 3.4. We have

$$
f_{3}(q)=\sum_{\substack{n \geq 0 \\|j| \leq n}}(-1)^{j} q^{2 n^{2}+2 n-j^{2}}
$$

Proof. We use the Bailey pair from Theorem 3.3 with $q \rightarrow q^{2}, b=-q, c=-1$, and $a=q^{2}$ in Bailey's Lemma with $\rho_{1}=q$ and $\rho_{2}=q^{2}$. The " $\beta$-side" equals

$$
\frac{1}{1+q} \sum_{n=0}^{\infty} \frac{\left(q, q^{2} ; q^{2}\right)_{n} q^{n}}{\left(-q^{3},-q^{2} ; q^{2}\right)_{n}}=\sum_{n=0}^{\infty} \frac{(q)_{2 n}}{(-q)_{2 n+1}} q^{n}=f_{3}(q)
$$

The " $\alpha$-side" is

$$
\sum_{n=0}^{\infty} q^{2 n^{2}+2 n}\left(1+2 \sum_{j=1}^{n}(-1)^{j} q^{-j^{2}}\right)
$$

which is the right-hand side of (3.3).

Proof of Theorem 1.3. Using Proposition 3.4 we get

$$
q f_{3}\left(q^{2}\right)=\sum_{\substack{n \geq 0 \\|j| \leq n}}(-1)^{j} q^{(2 n+1)^{2}-2 j^{2}}
$$

We write each ideal $\mathbf{a}$ as $\mathbf{a}=(u+v \sqrt{2})$ as in the proof of Theorem 1.1. Note that the Kronecker symbol implies that only elements with $u$ odd survive and that $(-1)^{v}=\left(\frac{-4}{\mathcal{N}(\mathbf{a})}\right)$. This finishes the proof of Theorem 1.3.

We now write $f_{4}(q)$ as a Hecke-type sum.

Proposition 3.5. We have

$$
f_{4}(q)=-\sum_{\substack{n \geq 0 \\-n-1 \leq j \leq n}}(-1)^{j} q^{2 n^{2}+4 n+2-j^{2}} .
$$

Proof. We use the Bailey pair from Theorem 3.3 with $q \rightarrow q^{2}, b=-q, c=-q^{2}$, and $a=q^{4}$ in Bailey's Lemma with $\rho_{1}=q^{2}$ and $\rho_{2}=q^{3}$. The " $\beta$-side" then equals

$$
\frac{1-q}{1-q^{4}} \sum_{n=0}^{\infty} \frac{\left(q^{2}, q^{3} ; q^{2}\right)_{n}}{\left(-q^{3},-q^{4} ; q^{2}\right)_{n}} q^{n}=\frac{1}{1-q} \sum_{n=0}^{\infty} \frac{(q)_{2 n+1}}{(-q)_{2 n+2}} q^{n}=\frac{q^{-1}}{1-q} f_{4}(q) .
$$


The " $\alpha$-side" equals

$$
\begin{aligned}
\sum_{n=0}^{\infty} q^{2 n^{2}+4 n}(1+ & \left.\frac{q}{1-q} \sum_{j=1}^{n}(-1)^{j}\left(q^{-j^{2}-2 j-1}-q^{-j^{2}}\right)\right) \\
& =-\frac{q}{1-q} \sum_{n=0}^{\infty} q^{2 n^{2}+4 n} \sum_{j=-n-1}^{n}(-1)^{j} q^{-j^{2}}
\end{aligned}
$$

Multiplying with $q(1-q)$ gives Proposition 3.5 .

Proof of Theorem 1.4. By Proposition 3.5, we have

$$
-f_{4}\left(q^{2}\right)=\sum_{\substack{n \geq 0 \\-n-1 \leq j \leq n}}(-1)^{j} q^{(2 n+2)^{2}-2 j^{2}} .
$$

Using Lemma 3 of [3], we write each ideal as $\mathbf{a}=(u+\sqrt{2} v)$ with $\mathcal{N}(\mathbf{a})=2 v^{2}-u^{2}$ with $v>0$ and $-v<u \leq v$. As before this yields

$$
f_{4}\left(q^{2}\right)=-\sum_{\substack{\mathbf{a} \subset O_{K} \\ 2 \mid \mathcal{N}(\mathbf{a})}}(-1)^{\frac{\mathcal{N}(\mathbf{a})}{2}} q^{\mathcal{N}(\mathbf{a})} .
$$

To finish the proof, we note that there is a unique ideal of norm 2 , namely $(\sqrt{2})$ of $O_{K}$ which gives Theorem 1.4.

As before, the main step will be to rewrite $f_{5}$ using Bailey's Lemma.

Proposition 3.6. We have

$$
f_{5}(q)=\sum_{\substack{n \geq 0 \\|j| \leq n}} q^{\frac{3\left(n^{2}+n\right)}{2}-\frac{j^{2}+j}{2}} .
$$

Proof. We use Theorem 3.3 with $b=q^{1 / 2}, c=-q^{1 / 2}$, and $a=q$ in Bailey's Lemma with $\rho_{1}=q$ and $\rho_{2} \rightarrow \infty$. The " $\beta$-side" becomes

$$
\frac{1}{1-q} \sum_{n=0}^{\infty}(-1)^{n} \frac{(q)_{n}}{\left(q^{3} ; q^{2}\right)_{n}} q^{\frac{n^{2}+n}{2}}=f_{5}(q)
$$

The " $\alpha$-side" equals

$$
\sum_{n=0}^{\infty} q^{\frac{3\left(n^{2}+n\right)}{2}}\left(1+\sum_{j=1}^{n} q^{-\frac{j^{2}+j}{2}}\left(1+q^{j}\right)\right),
$$

which is the right-hand side of Proposition 3.6

Proof of Theorem 1.5. It follows easily by Proposition 3.6 that

$$
q^{2} f_{5}\left(q^{8}\right)=\sum_{\substack{n \geq 0 \\|j| \leq n}} q^{3(2 n+1)^{2}-(2 j+1)^{2}} .
$$

Using Lemma 3 of [3], we write $\mathbf{a}=(u+\sqrt{3} v)$ with $\mathcal{N}(\mathbf{a})=3 v^{2}-u^{2}, v>0$, and $-v<u \leq v$. This yields as before

$$
q^{2} f_{5}\left(q^{8}\right)=\sum_{\substack{\mathbf{a} \subset O_{L} \\ \mathcal{N}(\mathbf{a}) \equiv 2}} q^{\mathcal{N}(\mathbf{a})}
$$


The theorem then follows after dividing by the unique ideal $(1+\sqrt{3})$ of $O_{L}$ of norm 2.

Proposition 3.7. We have

$$
f_{6}(q)=-\sum_{\substack{n \geq 0 \\|j| \leq n}} q^{3 n^{2}+3 n+1-j^{2}}-\sum_{\substack{n \geq 0 \\-n \leq j \leq n-1}} q^{3 n^{2}-j^{2}-j} .
$$

Proof. We take the Bailey pair from Lemma 12 of [1] with respect to $a=1$ with

$$
\begin{aligned}
& \mathcal{B}_{0}=\mathcal{B}_{0}(q):=0 \\
& \mathcal{B}_{n}=\mathcal{B}_{n}(q):=\frac{1}{\left(q^{n}\right)_{n}}=\frac{(q)_{n-1}}{(q)_{2 n-1}}
\end{aligned}
$$

and

$$
\begin{aligned}
\mathcal{A}_{2 n} & =\mathcal{A}_{2 n}(q):=-q^{3 n^{2}-2 n}\left(1-q^{4 n}\right) \sum_{j=-n}^{n-1} q^{-j^{2}-j}, \\
\mathcal{A}_{2 n+1} & =\mathcal{A}_{2 n+1}(q):=q^{3 n^{2}+n}\left(1-q^{4 n+2}\right) \sum_{|j| \leq n} q^{-j^{2}} .
\end{aligned}
$$

We take in Bailey's Lemma $\rho_{1}=-1$, divide on both sides by $2\left(1-\rho_{2}\right)$, and then let $\rho_{2} \rightarrow 1$. On the " $\beta$-side" we then have

$$
\sum_{n=1}^{\infty}(-1)^{n} \frac{(-q)_{n-1}(q)_{n-1}^{2}}{(q)_{2 n-1}}=f_{6}(q)
$$

The " $\alpha$-side" equals

$$
\sum_{n=1}^{\infty} \frac{q^{2 n}}{1-q^{4 n}} \mathcal{A}_{2 n}-\sum_{n \geq 0} \frac{q^{2 n+1}}{1-q^{4 n+2}} \mathcal{A}_{2 n+1} .
$$

Inserting (3.4) and 3.5 gives Proposition 3.7.

Proof of Theorem [1.6. From Proposition 3.7 we have

$$
q^{-1} f_{6}\left(q^{4}\right)=-\sum_{\substack{n \geq 0 \\|j| \leq n}} q^{3(2 n+1)^{2}-(2 j)^{2}}-\sum_{\substack{n \geq 0 \\-n \leq j \leq n-1}} q^{3(2 n)^{2}-(2 j+1)^{2}}
$$

From this we immediately conclude the theorem as before.

We move on to the corresponding proposition for $f_{7}$.

Proposition 3.8. We have

$$
f_{7}(q)=\sum_{\substack{n \geq 0 \\|j| \leq n}}(-1)^{n+j} q^{3 n^{2}+2 n-j^{2}}\left(1-q^{2 n+1}\right) .
$$

Proof. We use Theorem 3.3 with $q \mapsto q^{2}, b=-1, c=-q$, and $a=q^{2}$ in Bailey's Lemma with $\rho_{1}=q^{2}$ and $\rho_{2} \rightarrow \infty$. The " $\beta$-side" is

$$
\frac{1}{1-q^{2}} \sum_{n=0}^{\infty} \frac{\left(q^{2} ; q^{2}\right)_{n}(-1)^{n} q^{n(n+1)}}{\left(-q^{2},-q^{3} ; q^{2}\right)_{n}}=\frac{1}{1-q} \sum_{n=0}^{\infty} \frac{\left(q^{2} ; q^{2}\right)_{n}(-1)^{n} q^{n(n+1)}}{(-q)_{2 n+1}}=\frac{f_{7}(q)}{1-q}
$$

The " $\alpha$-side" equals the right-hand side of Proposition 3.8 divided by $1-q$, giving Proposition 3.8 . 
Proof of Theorem 1.7. From Proposition 3.8 we immediately obtain

$$
q f_{7}\left(q^{3}\right)=\sum_{\substack{n \geq 0 \\|j| \leq n}}(-1)^{n+j}\left(q^{(3 n+1)^{2}-3 j^{2}}-q^{(3 n+2)^{2}-3 j^{2}}\right) .
$$

We use Lemma 3 of $\left[3\right.$ to write $\mathbf{a}=(u+\sqrt{3} v)$ with $\mathcal{N}(\mathbf{a})=u^{2}-3 v^{2}, u>0$, $-\frac{u}{3}<v \leq \frac{u}{3}$. The condition $\mathcal{N}(\mathbf{a}) \equiv 1(\bmod 3)$ translates into the two summands of (3.6), noting that

$$
(-1)^{n+j}=-(-1)^{(3 n+1)^{2}-3 j^{2}}=(-1)^{(3 n+2)^{2}-3 j^{2}} .
$$

Finally, we show that $f_{8}$ may be written as a Hecke-type sum.

Proposition 3.9. We have

$$
f_{8}(q)=-\sum_{\substack{n \geq 1 \\-n \leq j \leq n-1}} q^{6 n^{2}-2 n-2 j^{2}-2 j}\left(1+q^{4 n}\right)+\sum_{\substack{n \geq 0 \\|j| \leq n}} q^{6 n^{2}+4 n+1-2 j^{2}}\left(1+q^{4 n+2}\right) .
$$

Proof. We use the Bailey pair $\mathcal{B}_{n}\left(q^{2}\right)$ and $\mathcal{A}_{n}\left(q^{2}\right)$ from the proof of Theorem 1.6. We then let in Bailey's Lemma $\rho_{1}=q$, divide both sides by $1-\rho_{2}$ and take $\rho_{2} \rightarrow 1$. The " $\beta$-side" gives

$$
\sum_{n=1}^{\infty} \frac{\left(q^{2} ; q^{2}\right)_{n-1}\left(q ; q^{2}\right)_{n}}{\left(q^{2 n} ; q^{2}\right)_{n}} q^{n}=\sum_{n=1}^{\infty} \frac{(q)_{2 n-1}}{\left(q^{2 n} ; q^{2}\right)_{n}} q^{n}=\sum_{n=1}^{\infty} \frac{(q)_{n-1}\left(q^{n}\right)_{n}}{\left(-q^{n}\right)_{n}\left(q^{n}\right)_{n}} q^{n}=f_{8}(q) .
$$

On the " $\alpha$-side" we have

$$
\sum_{n \geq 1} \frac{q^{n}}{1-q^{2 n}} \mathcal{A}_{n}\left(q^{2}\right)
$$

Proposition 3.9 then follows after using (3.4) and (3.5) to evaluate $\mathcal{A}_{n}\left(q^{2}\right)$.

Proof of Theorem 1.8, By Proposition 3.9, $q^{-2} f_{8}\left(q^{6}\right)$ equals

$$
\begin{aligned}
-\sum_{\substack{n \geq 0 \\
-n-1 \leq j \leq n}}\left(q^{(6 n+7)^{2}-3(2 j+1)^{2}}+q^{(6 n+5)^{2}-3(2 j+1)^{2}}\right) \\
\\
+\sum_{\substack{n \geq 0 \\
|j| \leq n}}\left(q^{(6 n+2)^{2}-3(2 j)^{2}}+q^{(6 n+4)^{2}-3(2 j)^{2}}\right) .
\end{aligned}
$$

As before we see that

$$
q^{-2} f_{8}\left(q^{6}\right)=\sum_{\mathcal{N}(\mathbf{a}) \equiv-2(\bmod 6)}(-1)^{\frac{\mathcal{N}(\mathbf{a})}{2}} q^{\mathcal{N}(\mathbf{a})}
$$

Theorem 1.8 then follows by dividing by the unique ideal $(1+\sqrt{3})$ of $O_{L}$ of norm 2 . 
3.1. Proof of the Corollories. Corollary 1.9 follows immediately from the Hecketype sums obtained in Section 3, using Theorem 1 of [17.

To prove Corollary 1.10, we note that in all cases the weightings only depended on the norm of $\mathbf{a}$, and hence the $n$-th coefficient in each case equals the number of ideals of norm $n$ times a weighting depending only on $n$. We next recall that unique factorization gives the number of ideals of $O_{K}$ and $O_{L}$ of norm $p^{\ell}$ as follows:

$$
\begin{aligned}
& \#\left\{\mathbf{a} \subset O_{K}: \mathcal{N}(\mathbf{a})=p^{\ell}\right\}=\left\{\begin{array}{lll}
\ell+1 & \text { if } p \equiv \pm 1 \quad(\bmod 8), \\
1 & \text { if } p \equiv \pm 3 \quad(\bmod 8) \text { and } \ell \text { is even }, \\
1 & \text { if } p=2, \\
0 & \text { if } p \equiv \pm 3 \quad(\bmod 8) \text { and } \ell \text { is odd },
\end{array}\right. \\
& \#\left\{\mathbf{a} \subset O_{L}: \mathcal{N}(\mathbf{a})=p^{\ell}\right\}=\left\{\begin{array}{lll}
\ell+1 & \text { if } p \equiv \pm 1 \quad(\bmod 12), \\
1 & \text { if } p \equiv \pm 5 \quad(\bmod 12) \text { and } \ell \text { is even }, \\
1 & \text { if } p=2,3, \\
0 & \text { if } p \equiv \pm 5 \quad(\bmod 12) \text { and } \ell \text { is odd }
\end{array}\right.
\end{aligned}
$$

Multiplicativity then gives an exact formula for the number of elements of norm $n$, based on the factorization. The proof of Corollary 1.10 now follows easily using the explicit form of the weighting.

\section{Combinatorial interpretations}

4.1. Theorem 1.1, Recalling the definition

$$
f_{1}(x ; q)=\frac{1}{1-q}+\sum_{n=1}^{\infty} \frac{q^{\frac{n^{2}+n}{2}}}{(x q)_{n}\left(1-x^{2} q^{2 n+1}\right)},
$$

we have $f_{1}(q)=f_{1}(-1 ; q)$. Hence we can give the combinatorial interpretation for $f_{1}(x ; q)$ and then plug in $x=-1$ to obtain the desired combinatorics for $f_{1}(q)$. We will see that the coefficient of $x^{r} q^{m}$ in $f_{1}(x ; q)-q$ is the number of partitions of $m$ with $r_{1}(\lambda)=r$.

For the term $\frac{1}{1-q}$ we take the partition $\lambda$ having only parts of size 1. It is clear in this case that $r_{1}(\lambda)=0$ in all cases. For $n \geq 1$, we form a triangle with sides of length $n$ in the upper left-hand corner of the Ferrer's diagram (with rank 0). For the power $x^{2 k} q^{k(2 n+1)}$ arising from $\frac{1}{1-x^{2} q^{2 n+1}}$, we then add $2 k$ to the first $n-1$ parts, add a part of size $2 k$, and $k$ parts of size 1 . If $k \geq 1$, then the resulting partition has repeated parts of size 1. Since the partition without the parts of size 1 still has precisely $n$ parts and the largest part is increased by $2 k$, the rank is increased by precisely $2 k$. Finally, $\frac{1}{(x q)_{n}}$ adds parts of size at most $n$, which we place along the "diagonal", each such part adding exactly one to $r_{1}(\lambda)$. The only repeated partition from our construction is the single part of size 1 , which we have subtracted.

\subsection{Theorem 1.2. Let}

$$
f_{2}(x ; q):=\frac{q}{1-q}+\sum_{n \geq 2} \frac{q^{\frac{n^{2}+n}{2}}}{(x q)_{n-1}\left(1-x^{2} q^{2 n-1}\right)} .
$$

We will see that the coefficient of $x^{r} q^{m}$ in $f_{2}(x ; q)$ is the number of partitions of $m$ with $r_{2}(\lambda)=r$. Then taking $x=-1$ gives the desired interpretation for $f_{2}(q)=f_{2}(-1 ; q)$. 
We begin by forming a triangle with sides of length $n$. We then append $2 k$ to the largest $n-1$ parts and append $k$ to the smallest part, where $k$ is the power of $x^{2} q^{2 n-1}$ coming from $\frac{1}{1-x^{2} q^{2 n-1}}$. The smallest part is now $k+1$, and if $n \neq 1$, then the rank is $2 k$ and the second smallest part is $2 k+2$. Finally, $\frac{1}{(x q)_{n-1}}$ adds parts of size at most $n-1$, which we place along the diagonal, adding one to $r_{2}(\lambda)$ for each part. The size of the smallest part is not changed in the final step, so our construction yields a bijection to $\lambda \in P_{2}$ with $n$ parts and smallest part $k+1$, having the desired rank if $n \neq 1$, while clearly if $n=1$, then $r_{2}(\lambda)=0$ by definition.

4.3. Theorem 1.3, We first define

$$
f_{3}(x ; q):=\sum_{n=0}^{\infty} \frac{\left(-x^{-1} q\right)_{n}}{\left(x^{-1} q\right)_{n}} x^{-1} q^{n} \cdot \frac{\left(-x q^{n+1}\right)_{n}}{\left(x q^{n+1}\right)_{n+1}} .
$$

For $\Lambda=(\mu, \lambda) \in P_{3}$, the power of $x$ will be used to count $r_{3}(\Lambda)$, the size of the largest part of $\lambda$ minus the number of parts in $\mu$.

It is clear that the coefficient of $x^{-r_{1}} q^{m_{1}}$ in $\frac{\left(-x^{-1} q\right)_{n}}{\left(x^{-1} q\right)_{n}} x^{-1} q^{n}$ is the number of overpartitions of $m_{1}$ with exactly $r_{1}$ parts and largest part $n$ (which is not overlined). We thus write $\Lambda \in P_{3}$ by $\Lambda=(\mu, \lambda)$, and the first component $\mu$ will be determined by this factor.

We next consider the second component $\lambda$ of the overpartition pair. For $x^{k} q^{k(n+1+r)}$ chosen from $\frac{\left(-x q^{n+1}\right)_{n}}{\left(x q^{n+1}\right)_{n+1}}$, we add $k$ parts of size $n+1$ and $k$ parts of size $r$ (when $r>0$ ). When choosing from the numerator, we underline the first occurrence of the part $r$ if $r>0$ and, if $r=0$, then we underline the first occurrence of the part $n+1$. Thus the coefficient of $x^{r_{2}} q^{m_{2}}$ in $\frac{\left(-x q^{n+1}\right)_{n}}{\left(x q^{n+1}\right)_{n+1}}$ counts the number of overpartitions of $m_{2}$ with largest part $n+1$, where each occurrence of a part of size at most $n$ is paired with a part of size $n+1$, and the number of occurrences of the part size $n+1$ equals $r_{2}$.

Combining these implies that the coefficient of $x^{r} q^{m}$ in $f_{3}(x ; q)$ is the number of overpartition pairs $\Lambda \in P_{3}$ with $|\Lambda|=m$ and $r_{3}(\lambda)=r$. We have $f_{3}(q)=$ $-f_{3}(-1 ; q)$, so that plugging in $x=-1$ gives us the generating function for those with $r_{3}(\Lambda)$ odd minus those with $r_{3}(\Lambda)$ even.

4.4. Theorem 1.4. To see that $f_{4}(q)$ generates the desired partitions, we proceed similarly as for $f_{3}$ and write

$$
f_{4}(x ; q):=\sum_{n=1}^{\infty} \frac{(-x q)_{n}}{(x q)_{n}} x q^{n} \cdot \frac{\left(-x q^{n+1}\right)_{n-1}}{\left(x q^{n+1}\right)_{n}} .
$$

We interpret $\frac{\left(-x^{-1} q\right)_{n}}{\left(x^{-1} q\right)_{n}} x^{-1} q^{n}$ as in the case for $f_{3}$. The factor $\frac{\left(-x q^{n+1}\right)_{n-1}}{\left(x q^{n+1}\right)_{n}}$ gives the generating function (in $x^{r_{2}} q^{m_{2}}$ ) for overpartitions of $m_{2}$ with largest part $n$ occuring $r_{2}$ times, where parts appear in pairs $(n, r)$ for some $1 \leq r \leq n$. Terms from the numerator have $1 \leq r<n$, and we overline the first occurrence of $r$. From this one easily sees that $f_{4}(x ; q)$ enumerates the claimed partitions.

4.5. Theorem 1.5. Recall that $\ell_{i}=\lambda_{i}-\lambda_{i+1}(1 \leq i \leq n-1)$ and $\ell_{n}=\lambda_{n}$, $E:=E_{\lambda}:=\left\{2 \leq r \leq n: \ell_{r}\right.$ is even $\}$ and $e:=\# E$. The second largest part of $\lambda$ (if it exists) must be at least $n-1+e$ because the size of the second largest part is $\lambda_{2}=\sum_{i=2}^{n} \ell_{i}$. Thus, it is natural to define

$$
d_{\lambda, 1}:=\lambda_{2}-((n-1)+e) .
$$


Since

$$
d_{\lambda, 1}=\left(\sum_{i=2}^{n} \ell_{i}\right)-(n-1+e)=\sum_{i \in E}\left(\ell_{i}-2\right)+\sum_{i \notin E, i>1}\left(\ell_{i}-1\right)
$$

is even, we may furthermore define the integral metric $d_{\lambda}:=\frac{d_{\lambda, 1}}{2}$ alluded to in the introduction.

We now rewrite

$$
f_{5}(q)=\frac{1+q^{2}}{1-q^{3}}+\sum_{n=2}^{\infty}(-1)^{n} \frac{\left(q^{2}\right)_{n-1}}{\left(q^{3} ; q^{2}\right)_{n}} q^{\frac{n^{2}+n}{2}} .
$$

The term

$$
\frac{1+q^{2}}{1-q^{3}}=\sum_{j=0}^{\infty}\left(q^{3 j}+q^{3 j+2}\right)
$$

corresponds to partitions in $P_{5}$ with exactly one part which is not congruent to 1 modulo 3 .

For $n \geq 2$, we first form a triangle with sides of length $n$ weighted by the largest part. We adjoin $3 k_{r}$ to the largest part and $2 k_{r}$ to the next $r-1$ parts, where $k_{r}$ is the power of $q^{2 r+1}$ coming from $\frac{1}{\left(q^{3} ; q^{2}\right)_{n}}$. At this stage of the construction we have added an even number to each part other than the largest, so $\ell_{i}$ is odd for $i>1$ and hence $E=\emptyset$. Notice that $d_{\lambda}=\sum_{r=2}^{n} k_{r}$ and $\ell_{1}=3 k_{1}+d_{\lambda}+1$. Hence we have thusfar constructed all $\lambda \in P_{5}$ with $E=\emptyset, n$ parts, and the desired weighting.

Finally we add 1 to the first $r$ parts if $q^{r}$ is chosen from $\left(q^{2}\right)_{n-1}$. Notice that $n$ represents the number of parts and $r \in E$ if and only if we chose $q^{r}$ in the final step. Moreover the overall weighting is

$$
(-1)^{n+e}=(-1)^{n+e+2 d_{\lambda}}=-(-1)^{\lambda_{2}} .
$$

\subsection{Theorem 1.6. Define}

$$
f_{6}(x ; q):=\sum_{n=1}^{\infty} \frac{\left(-x^{-1} q^{2} ; q^{2}\right)_{n-1}}{\left(q^{n}\right)_{n}} x^{n} q^{n} .
$$

In this case the power of $x$ will count the largest part minus the number of overlined parts.

We begin with a part of size $n$ from the factor $x^{n} q^{n}$. We next add $k$ parts of size $n$ and $k$ parts of size $r$ (if $r>0$ ), where $k$ is the power of $q^{n+r}$ coming from $\frac{1}{\left(q^{n}\right)_{n}}$. We finally add two parts of size $r$ and overline the first occurrence of $r$ if $q^{2 r}$ is chosen from $\left(-x^{-1} q^{2} ; q^{2}\right)_{n-1}$. Since all of the parts other than the ones coming from $\frac{1}{1-q^{n}}$ occur in pairs with one of the parts either overlined or equal to $n$, we obtain an overpartition in $P_{6}$ with the correct power of $x$.

\subsection{Theorem 1.7, First define}

$$
f_{7}(x ; q):=\frac{1}{1-x q}+\sum_{n=1}^{\infty} \frac{x^{n} q^{n^{2}+n}(-x q)_{n}}{\left(x q^{n+1}\right)_{n+1}} .
$$

Here $x$ will count the statistic of the number of parts.

The term $x^{r} q^{r}$ in $\frac{1}{1-x q}$ corresponds to partitions with $r$ parts all equal to one.

For $n \geq 1$ we first place $n$ parts of size $n+1$. We then append $k$ to the first $n$ parts and add $k$ parts of size $r$, where $k$ is the power of $x q^{n+r}$ chosen from $\frac{1}{\left(-q^{n+1}\right)_{n+1}}$ (note that we have added $k$ parts). Finally, $(-x q)_{n}$ corresponds to overlined parts 
of size at most $n$. Notice that $n=M(\lambda)$. Moreover, since the largest part is at least 2 , we have no overlap with the partitions coming from $\frac{1}{1-x q}$. This easily yields the desired bijection to $P_{7}$ with the power of $x$ counting the number of parts. Clearly $f_{7}(-1 ; q)=f_{7}(q)$, and we have the desired interpretation for $f_{7}$.

4.8. Theorem 1.8. Similarly to the above cases, we define

$$
f_{8}(x ; q):=x \sum_{n=1}^{\infty} \frac{\left(-x^{-1} q\right)_{n-1}}{\left(x q^{n}\right)_{n}} q^{n} .
$$

We start with a part of size $n$. For $x q^{r}$ chosen from $(-x q)_{n-1}$ we add an overlined part of size $r$. We finally add $k$ parts of size $n$ and $k$ parts of size $r$ (when $r>0$ ), where $k$ is the power of $x q^{n+r}$ occurring from $\frac{1}{\left(x q^{n}\right)_{n}}$. Thus, the power of $x^{r} q^{m}$ is the number of partitions $\lambda \in P_{8}$ of $m$ with $r_{8}(\lambda)=r$, and $f_{8}(q)=-f_{8}(-1 ; q)$ gives the desired result.

\section{ACKNOWLEDGEMENTS}

The authors thank Jeremy Lovejoy for helpful comments on earlier versions of this paper.

\section{REFERENCES}

[1] G. Andrews, The fifth and seventh order mock theta functions, Trans. Amer. Math. Soc. 293 (1986), pages 113-134. MR814916 (87f:33011)

[2] G. Andrews, $q$-series: Their development and application in analysis, number theory, combinatorics, physics, and computer algebra, CBMS Regional Conference Series in Mathematics, 66, Amer. Math. Soc., Providence, RI, 1986. MR858826 (88b:11063)

[3] G. Andrews, F. Dyson, and D. Hickerson, Partitions and indefinite quadratic forms, Invent. Math. 91 (1988), pages 391-407. MR928489 (89f:11071)

[4] G. Andrews and D. Hickerson, Ramanujan's "lost" notebook. VII. The sixth order mock theta functions, Adv. Math. 89 (1991), pages 60-105. MR.1123099 (92i:11027)

[5] G. Andrews, J. Jimenez-Urroz, and K. Ono, q-series identities and values of certain Lfunctions, Duke Math. J. 108 (2001), pages 395-419. MR.1838657 (2002e:11055)

[6] K. Bringmann and K. Ono The $f(q)$ mock theta function conjecture and partition ranks, Invent. Math. 165 (2006), pages 243-266. MR2231957 (2007e:11127)

[7] K. Bringmann and K. Ono, Dyson's ranks and Maass forms, Ann. of Math, 171 (2010), pages 419-449. MR2630043

[8] H. Cohen, q-identities for Maass waveforms, Invent. Math. 91 (1988), pages 409-422. MR928490 (89f:11072)

[9] D. Corson, D. Favero, K. Liesinger, and S. Zubairy, Characters and q-series in $\mathbb{Q}(\sqrt{2})$, J. Number Theory 107 (2004), pages 392-405. MR2072397 (2005c:11126)

[10] S. Corteel and J. Lovejoy, Overpartitions, Trans. Amer. Math. Soc. 356 (2004), pages 16231635. MR2034322 (2004j:11118)

[11] F. Dyson, Some guesses in the theory of partitions, Eureka (Cambridge) 8 (1944), pages $10-15$.

[12] J. Lovejoy, Lacunary partition functions, Math. Res. Lett. 9 (2002), pages 191-198. MR:1909637 (2003f:11157)

[13] J. Lovejoy, Overpartitions and real quadratic fields, J. Number Theory 106 (2004), pages 178-186. MR2049600 (2005b:11164)

[14] J. Lovejoy, Overpartition pairs, Ann. Inst. Fourier 56 (2006), pages 781-794. MR 2244229 (2008a:11128)

[15] J. Lovejoy and O. Mallet, n-color overpartitions, twisted divisor functions, and RogersRamanujan identities, South East Asian J. Math. Math. Sci. (Andrews' 70th birthday issue) 6 (2008), pages 23-36. MR2442094 (2009m:11169) 
[16] J. Lovejoy and K. Ono, Hypergeometric generating functions for values of Dirichlet and other L-functions, Proc. Nat. Acad. Sci. USA 100 (2003), pages 6904-6909. MR.1982655 (2004h:33037)

[17] R. Odoni, On the norms of integers in a full module of an algebraic number field and the distribution of values of binary integral quadratic forms, Mathematica 22 (1975), pages 108111. MR0389856 (52:10686)

[18] S. Ramanujan, The lost notebook and other unpublished papers, Narosa, New Delhi, 1988. MR947735 (89j:01078)

[19] S. P. Zwegers, Mock theta-functions and real analytic modular forms, q-series with applications to combinatorics, number theory, and physics (Urbana, IL, 2000), 269-277, Contemp. Math., 291, Amer. Math. Soc., Providence, RI, 2001. MR.1874536 (2003f:11061)

Mathematical Institute, University of Cologne, Weyertal 86-90, 50931 Cologne, GERMANY

E-mail address: kbringma@math.uni-koeln.de

Department of Mathematics, Radboud University, Postbus 9010, 6500 GL, Nijmegen, NETHERLANDS

Current address: Mathematical Institute, University of Cologne, Weyertal 86-90, 50931 Cologne, Germany

E-mail address: bkane@mi.uni-koeln.de 